In der Rubrik „Literatur kompakt" werden die wichtigsten Originalarbeiten aus der internationalen Fachliteratur referiert.

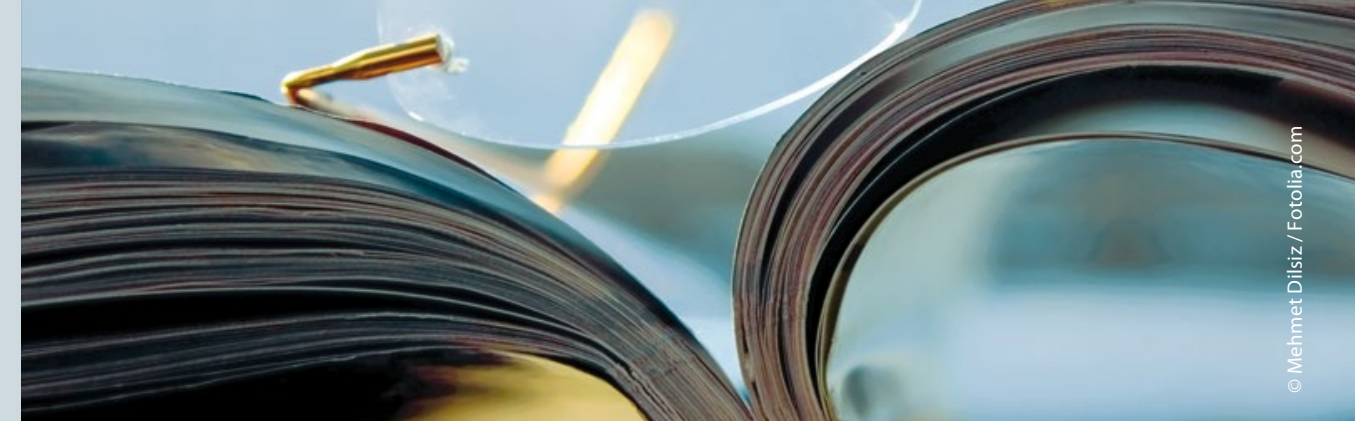

\section{Nach radikaler Zystektomie PSA-Wert kontrollieren}

\begin{abstract}
Bei mehr als $50 \%$ der Männer, die sich wegen eines Harnblasenkarzinoms einer radikalen Zystektomie unterziehen, wird einer österreichischen Studie zufolge auch ein Prostatakarzinom entdeckt. Wegen des hohen Risikos für Rezidive und Metastasen des Prostatatumors halten die Studienautoren deshalb PSA-Kontrollen nach solchen Eingriffen für erforderlich.
\end{abstract}

V or allem bei jüngeren Männern wird eine Zystektomie heute in den meisten Fällen prostataschonend durchgeführt, um die Miktion, aber auch die sexuelle Funktion des Patienten nicht zu beeinträchtigen. Solche schonenden Techniken können jedoch ein erhöhtes Risiko für weitere Tumorherde in sich bergen.

Im Rahmen einer retrospektiven Analyse haben Dr. Isabell Heidegger von der Medizinischen Universität Innsbruck, Österreich, und Kollegen anhand der Gewebeproben die Häufigkeit eines Prostatakarzinoms nach standardmäßiger, radikaler Zystektomie bei Männern wegen eines fortgeschrittenen Blasenkarzinoms untersucht. Die Urologen verfolgten darüber hinaus über zehn Jahre lang retrospektiv die Daten der 213 Patienten ohne chemotherapeutische Vorbehandlung. Bei 53,1 \% der Patienten konnten Heidegger et al. bei der histologischen Untersuchung nach der radikalen Zystektomie ein Prostatakarzinom feststellen. Als dessen signifikante Prädiktoren erwiesen sich das Patientenalter, der Gesamt-PSA-Wert sowie der Anteil des freien PSA im Blut (fPSA).

Patienten mit begleitendem Prostatakarzinom waren im Mittel 71 Jahre alt, während das Durchschnittsalter in der Gruppe ohne Zweittumoren bei 68 Jahren lag. Der durchschnittliche präoperative PSA-Wert lag bei Männern mit Prostatakarzinom bei $4,2 \mathrm{ng} / \mathrm{ml}$ und bei den Probanden ohne Prostatakrebs bei $1,6 \mathrm{ng} / \mathrm{ml}$. Ähnlich signifikante Unterschiede ergaben sich für das fPSA vor der radikalen Zystektomie mit 19,1\% versus $24,6 \%$. Bei 63,7\% der Prostatakarzinompatienten fand sich ein ungünstiger Histologiebefund der Harnblase ( $\geq$ pT3), bei 52,2\% der Männer lag der Gleason Score (GS) bei $\geq 7$ und bei $10,6 \%$ der Patienten hatte der Prostatakrebs die Organgrenze überschritten $(\geq$ pT3a).

Schließlich analysierten Heidegger und ihre Kollegen die Häufigkeit von Rezidiven bei Patienten mit Prostatakarzinom und fortgeschrittenem Blasenkarzinom innerhalb des gesamten Studienzeitraums. Im ersten Jahr nach der radikalen Zystektomie wurde alle drei Monate der PSA-Wert kontrolliert, danach bis zum Ende des fünften Jahres zweimal und dann schließlich einmal jährlich.

Die 5,7 \% der Patienten, bei denen der PSA-Wert auch sechs Wochen nach der radikalen Zystektomie noch erhöht war, wurden einer Radiotherapie unterzogen. Doch in der Regel sank der PSAWert bei den Patienten nach der Operation auf $<0,003 \mathrm{ng} / \mathrm{ml}$. 15 von 53 Männern erfuhren allerdings dennoch ein biochemisches Rezidiv ihres Prostatakarzinoms (PSA $\geq 0,2 \mathrm{ng} / \mathrm{ml}$ ). 86,7 \% von ihnen hatten einen GS $\geq 7$ und bei $80 \%$ der Probanden mit biochemischem Rezidiv zeigte die histologische Untersuchung, dass sich das Blasenkarzinom zum Zeitpunkt der Operation bereits über die Harnblase hinaus ausgebreitet hatte $(\geq \mathrm{pT} 3)$.

$40 \%$ der Patienten mit Prostatakarzinomrezidiv verstarben letztlich innerhalb von neun Monaten bis vier Jahren nach der Zystektomie nicht an ihrem Blasen-, sondern an ihrem Prostatakarzinom.

Fazit: Laut Heidegger und ihren Kollegen ist ein ungünstiger Histologiebefund der Harnblase ein Risikofaktor sowohl für ein Prostatakarzinom als auch für ein biochemisches PSA-Rezidiv. Den Ergebnissen ihrer Studie zufolge sind die österreichischen Urologen der Ansicht, dass eine prostatasparende Zystektomie nur sehr ausgewählten Patienten ohne primäre Beteiligung der prostatischen Harnröhre und auch nur nach präoperativem Ausschluss eines Prostatakarzinoms durch digital-rektale Untersuchung und PSA-Bestimmung angeboten werden sollte.

Dr. Christine Starostzik

Heidegger I et al. High incidence of clinically significant concomitant prostate cancer in patients undergoing radical cystectomy for bladder cancer: a 10-year single-center experience. Urol Oncol. 2016; pii: S1078-1439(16)30370-2; doi: 10.1016/j.urolonc.2016.11.004. 